\title{
Immediate Dentin Sealing with Self-etch Dentin Bonding Agent for Indirect Restoration
}

\author{
${ }^{1}$ Murillo M Leite, ${ }^{2}$ Dhiogo R de Souza, ${ }^{3}$ Marco A Carvalho, ${ }^{4}$ Lawrence G Lopes, ${ }^{5}$ Érica M de Torres
}

\begin{abstract}
This case report describes the immediate dentin sealing (IDS) technique with self-etch dentin bonding agent (DBA) for indirect restorations. To avoid problems related to dentin exposure when preparing teeth for indirect restorations, the sealing of the dentin immediately after preparation and before impression has been recommended. The IDS technique with self-etch DBA prevents the patient from inconveniences associated with dentin demineralization by phosphoric acid in the traditional total-etch technique. The case report presented describes the indirect restoration of the right upper first molar using the IDS technique with a self-etch DBA covered with a low-viscosity composite resin. The lithium disilicate glass ceramic restoration was obtained with a computer-aided design/computer-aided manufacturing (CAD/CAM) system. Selective enamel etching occurred before cementation with resin cement. A follow-up of 15 months revealed success of the treatment and lack of sensitivity. The incorporation of IDS with indirect restoring teeth adhesively may provide better marginal fit, reduce marginal infiltration, protect the dentin-pulp complex, reduce postoperative sensitivity, bring more comfort to the patient, and is long-lasting to the restored teeth.
\end{abstract}

Clinical significance: The IDS technique with a self-etch DBA is an excellent clinical option to avoid some inconveniences of traditional protocols for indirect restorations.

Keywords: Dentin bonding agents, Denture partial fixed, Resin cements.

How to cite this article: Leite MM, de Souza DR, Carvalho MA, Lopes LG, de Torres ÉM. Immediate Dentin Sealing with Selfetch Dentin Bonding Agent for Indirect Restoration. World J Dent 2017;8(6):490-495.

\section{Source of support: Nil}

Conflict of interest: None

\section{INTRODUCTION}

Considering the current state of the art of dental materials and minimally invasive techniques, additive procedures are always preferable when restoring teeth. Nevertheless,

1,2,4,5 Department of Oral Health, School of Dentistry, Federal University of Goiás, Goiânia, Goiás, Brazil

${ }^{3}$ Department of Prosthodontics and Periodontology, University of Campinas, Piracicaba, São Paulo, Brazil

Corresponding Author: Murillo M Leite, Department of Oral Health, School of Dentistry, Federal University of Goiás, Goiânia Goiás, Brazil, Phone: +5562984022800, e-mail: murillo_leite@ hotmail.com many times conservative procedures are not a viable option and dentin may be exposed during preparation. ${ }^{1}$ The exposed dentin tubules transmit mechanical and chemical stimulus and favor bacterial infiltration. ${ }^{2}$ Consequently, sensitivity may occur as well as irreversible pulp alterations. ${ }^{1-3}$

Conventionally, the use of adhesive system and resin cements for indirect restorations is only recommended after impression and temporization steps, in other words, at restoration delivery. ${ }^{1,4}$ As temporary materials do not provide adhesion to dentin, the tooth is exposed to external aggressions, such as saliva, impression material, temporary material, temporary cement, bacteria among others. ${ }^{1,2}$ Thus, sensitivity is more likely to occur, as well as pulp pain ${ }^{2}$ and weakened bond strength due to dentin contamination. ${ }^{1,4}$

The classical bonding technique consists in the application of phosphoric acid, adhesive system, and resin cement at the time of restoration delivery. The prepolymerization of the DBA before cementation may interfere on the fitting of the restoration. Hence, some studies recommend to avoid prepolymerization during cementation. ${ }^{1,4-6}$ On the contrary, the pressure of the restoration and cement during the seating on unpolymerized DBA may collapse the collagen fibril meshwork at the hybrid layer, affecting negatively the bond strength., ${ }^{1,4} 6$

Thus, to avoid problems related to dentin exposure when preparing teeth for indirect restorations, the sealing of the dentin immediately after preparation and before impression has been recommended..$^{1-9}$ The IDS technique is also known as prehybridization, resin coating, and dual bonding technique. ${ }^{10}$ According to the literature, this approach provides increased bond strength, ${ }^{1-8,10-15}$ less gap $^{12}$ formation, ${ }^{1,2,11}$ reduction on sensitivity, ${ }^{2,10-12}$ decreased marginal infiltration, ${ }^{2,5}$ and protection of the dentin against bacterial infiltration., ${ }^{1,5,6,10}$ It is important to outline that the freshly cut dentin is ideal for bonding procedures. $1,3,5,6$

There are some variations of the IDS technique regarding the adhesive system type and the additional use of a low-viscosity resin after DBA.

Regarding DBA, the use of systems that require pretreatment of the dentin with phosphoric acid is proven to be efficient once the protocol is strictly followed to obtain an efficient hybrid layer. ${ }^{1}$ The number of steps, 
time of etching, and the need for dentin to be humid when applying the primer (wet bonding) are some factors that increase the technical sensitivity of the total-etch approach. Any misuse during the process can lead to incomplete sealing of the dentin, culminating in postoperative sensitivity, recurrent decay, debonding, or fracture of indirect restoration. ${ }^{1}$

The self-etch technique is an alternative approach to avoid problems related to the total-etch steps, since it is less sensitive once there is no application of acid before infusing the dentin with the primer. The risk of collapse of the demineralized collagen fibers is decreased with the self-etch approach that leads to improvement in bond strength. 1,10,16,17 Contrasting the total-etch technique, the self-etch adhesives keep the smear layer as a substrate, incorporating it to the adhesive layer, what may reduce the postoperative sensitivity. ${ }^{10,16,17}$ Then, the hybrid layer may be covered with a low-viscosity composite resin layer to protect it during temporization and also dissipate stresses during occlusal function. ${ }^{11,12,18}$

Evidence-based dentistry aims to establish clinical conduct and treatment options that offer long-lasting results through the use of safe, simple, and minimally invasive techniques. Bearing this in mind, this case report aims to describe and discuss the IDS with self-etch DBA for indirect restoration of a tooth.

\section{CASE REPORT}

A 35-year-old woman seeks for dental treatment to change old restorations reported to be stained and worn off. After anamnesis, physical examination, and complementary examinations (periapical and bite-wing X-rays), an infiltrated extensive restoration with discoloration and altered anatomy on the upper right first molar was identified (Fig. 1).

The treatment plan proposed aimed to improve both oral function and esthetics of the patient with a self-etch
IDS approach associated with a CAD/CAM glass-ceramic partial restoration. The removal of the old restoration was performed with spherical diamond bur (KG Sorensen, São Paulo, Brazil) (Fig. 2A). After the failed restoration removal, rubber dam was placed for preparation cleaning with pumice and finishing under complete isolation. Clearfil SE Bond (Kuraray, Tokyo, Japan), a self-etch adhesive system, was chosen for IDS. The system consists of bottles: Self-etch primer and bond agents.

With the aid of a microbrush (KG Brush, KG Sorensen), the self-etch primer was applied on the freshly cut, clean, and dry dentin for 20 seconds, as recommended by the manufacturer (Fig. 2B). Then, the solvent was evaporated with a gentle air blow (Fig. 2C), and a bond layer was applied on the dentin infused by the primer (Fig. 2D). The bond layer was light-cured for 40 seconds with Emitter D (Schuster, Santa Maria, RS, Brazil) (Fig. 2E). A layer of flowable composite resin (Natural Look, DFL, Rio de Janeiro, Brazil) was applied on the sealed dentin with the aid of a calcium hydroxide applicator and light cured for 40 seconds (Fig. 2F).

Composite resin (Charisma, Heraeus Kulzer, Hanau, Germany) was used to smooth the internal edges and fill the undercuts of the preparation and light-cured for 40 seconds (Fig. 3A). For final light curing of the oxygeninhibited layer, a glycerin layer (KY, Johnson and Johnson, São Paulo, Brazil) was applied over the adhesive layer and light cured for 20 seconds. To remove all adhesive excess on the enamel surface, a fine diamond bur (KG Sorensen) was gently used to provide better enamel adhesion at the time of restoration delivery (Fig. 3B). Before impression, the preparation was cleaned with a $70 \%$ alcohol solution to avoid interactions with the impression material (Fig. 3C). The impression was carried out with polyvinyl siloxane (Futura AD, DFL, Rio de Janeiro, Brazil) using the two-step technique: Putty first as a preliminary impression, and then wash was used for fine details (Fig. 3D).
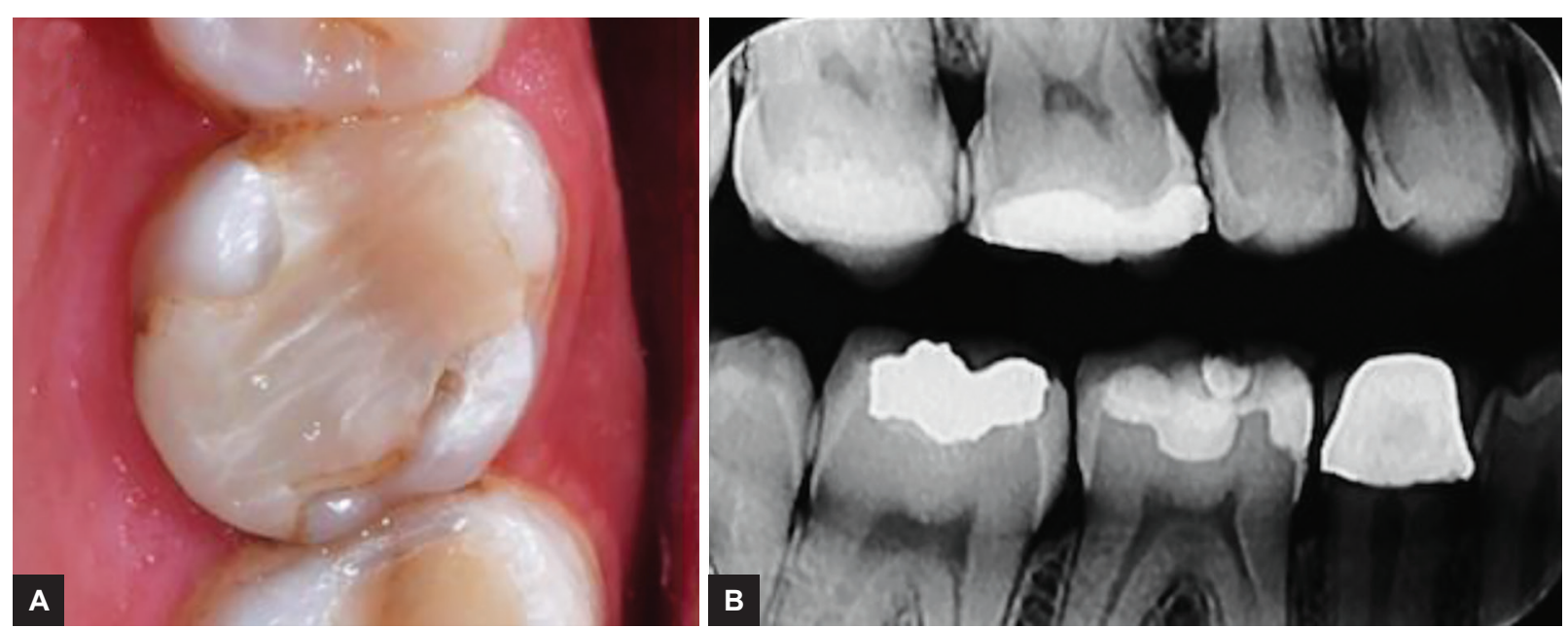

Figs $1 \mathrm{~A}$ and $\mathrm{B}$ : (A) Initial intraoral condition; and $(\mathrm{B})$ initial radiograph condition 

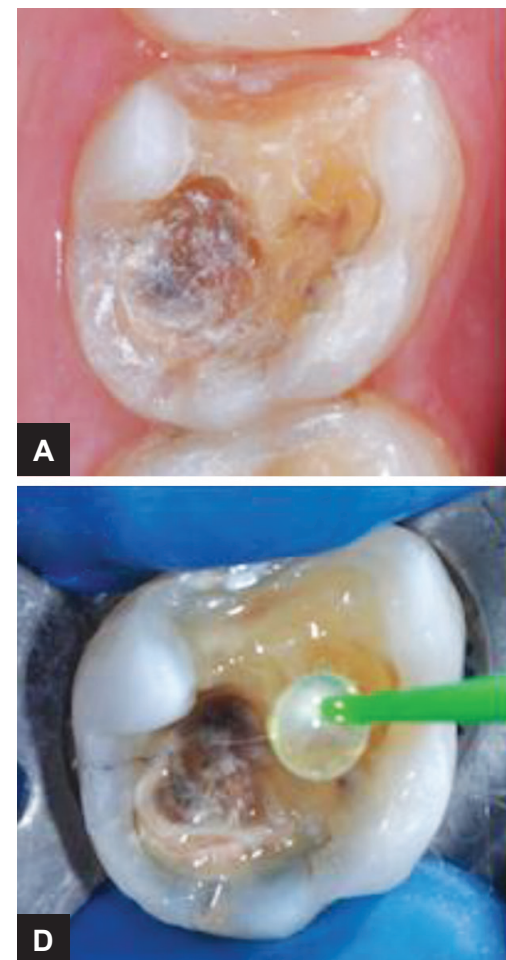

D
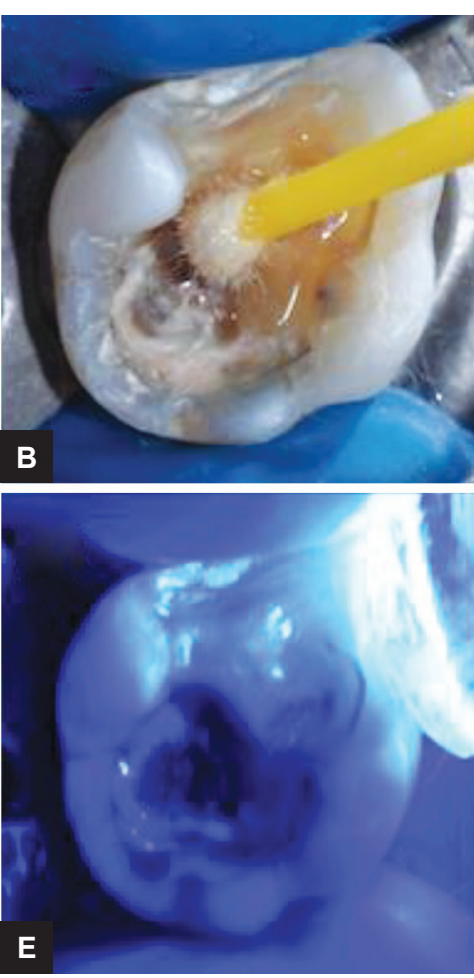
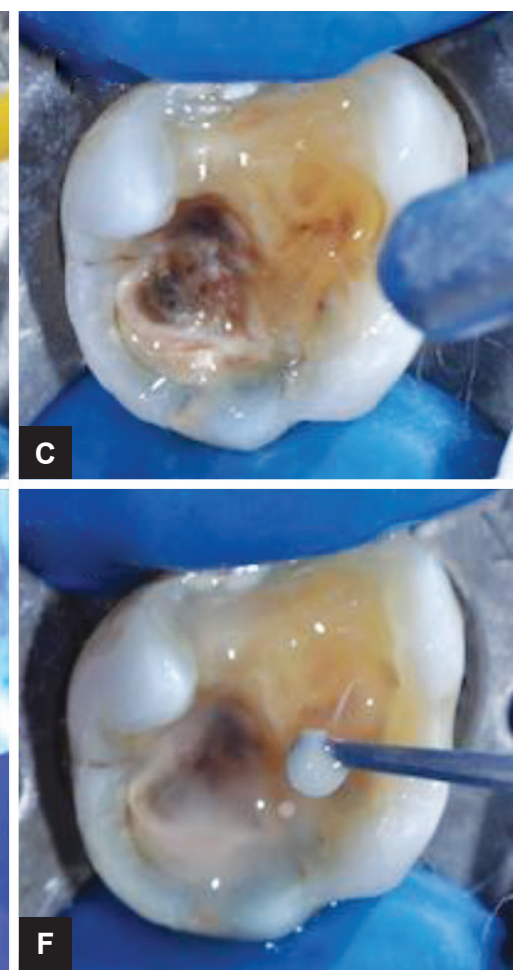

Figs 2A to F: (A) Intraoral condition immediately after restoration removal; (B) self-etch primer application for 20 seconds; (C) gentle air blowing for solvent evaporation; (D) bond layer application; (E) adhesive layer light curing for 40 seconds; and (F) flowable composite resin coat application
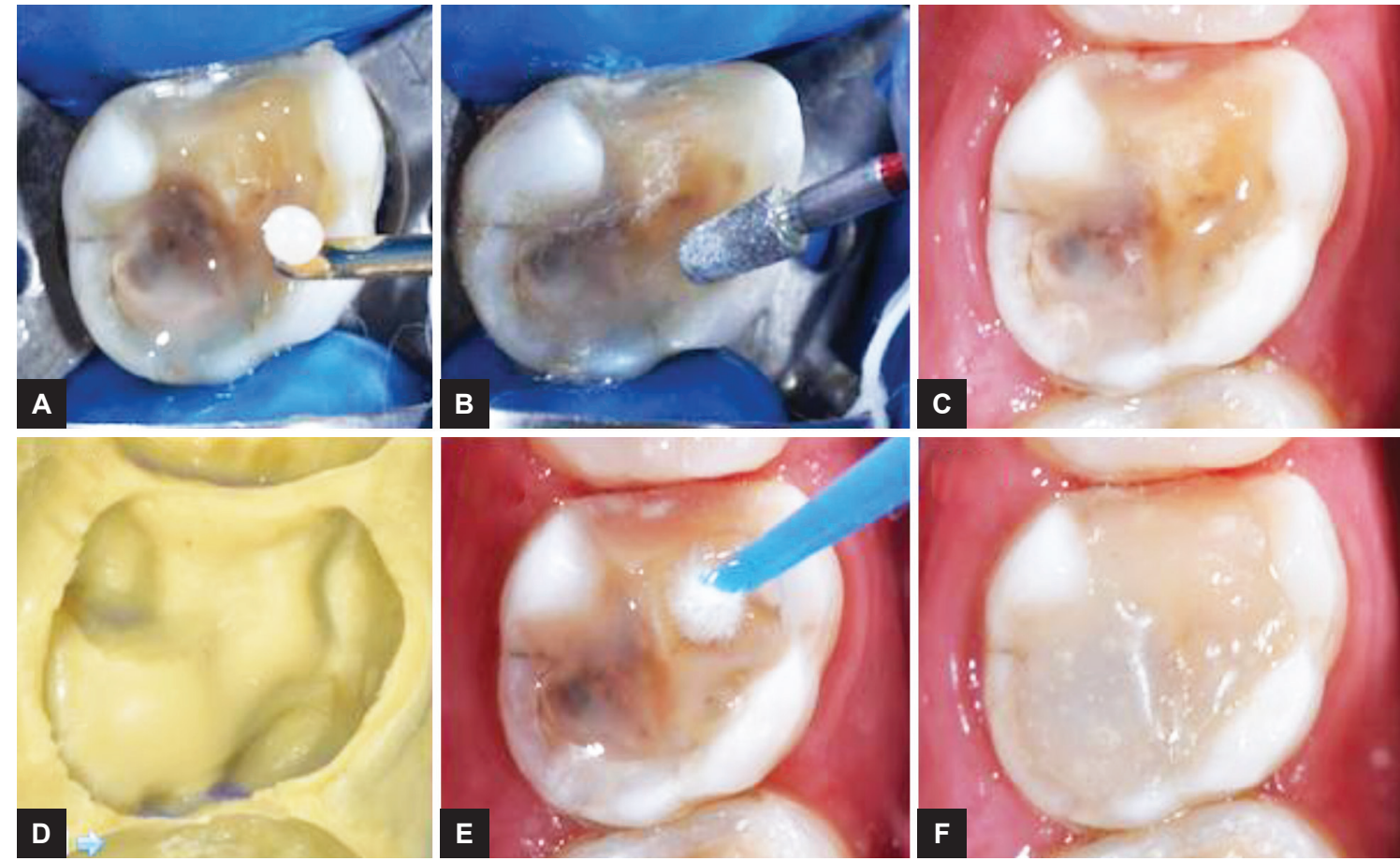

Figs $3 A$ to F: (A) Undercut filling with composite resin; (B) enamel margins finished with fine diamond bur; (C) preparation finished and ready for impression; (D) negative mold of the prepared tooth; (E) glycerin application; and $(\mathrm{F})$ temporary restoration

A thin layer of glycerin was applied to the preparation to avoid any adherence between the temporary restoration material (Bioplic, Biodinamica, Ibipora, Brazil) and the sealed dentin (Figs $3 \mathrm{E}$ and $\mathrm{F}$ ). The final ceramic restoration (IPS e.max CAD, Ivoclar Vivadent, Schaan, Germany) was performed using a CAD/CAM system (Cerec 4, Dentsply Sirona, New York, USA) (Figs 4A and B).

For final restoration, delivery anesthesia was not necessary, the temporary was removed, the try-in of the ceramic restoration was carried out to check fit, insertion 

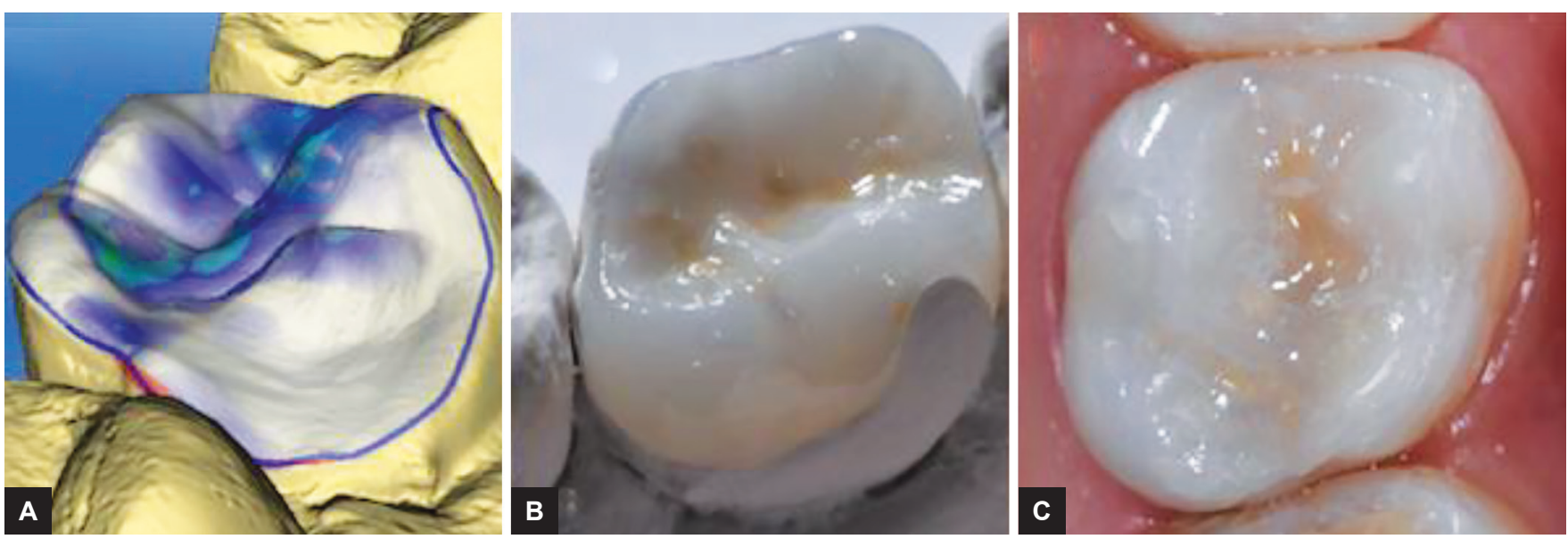

Figs 4A to C: (A) Virtual design of the restoration; (B) final restoration on stone model; and (C) final intraoral condition of the restored tooth

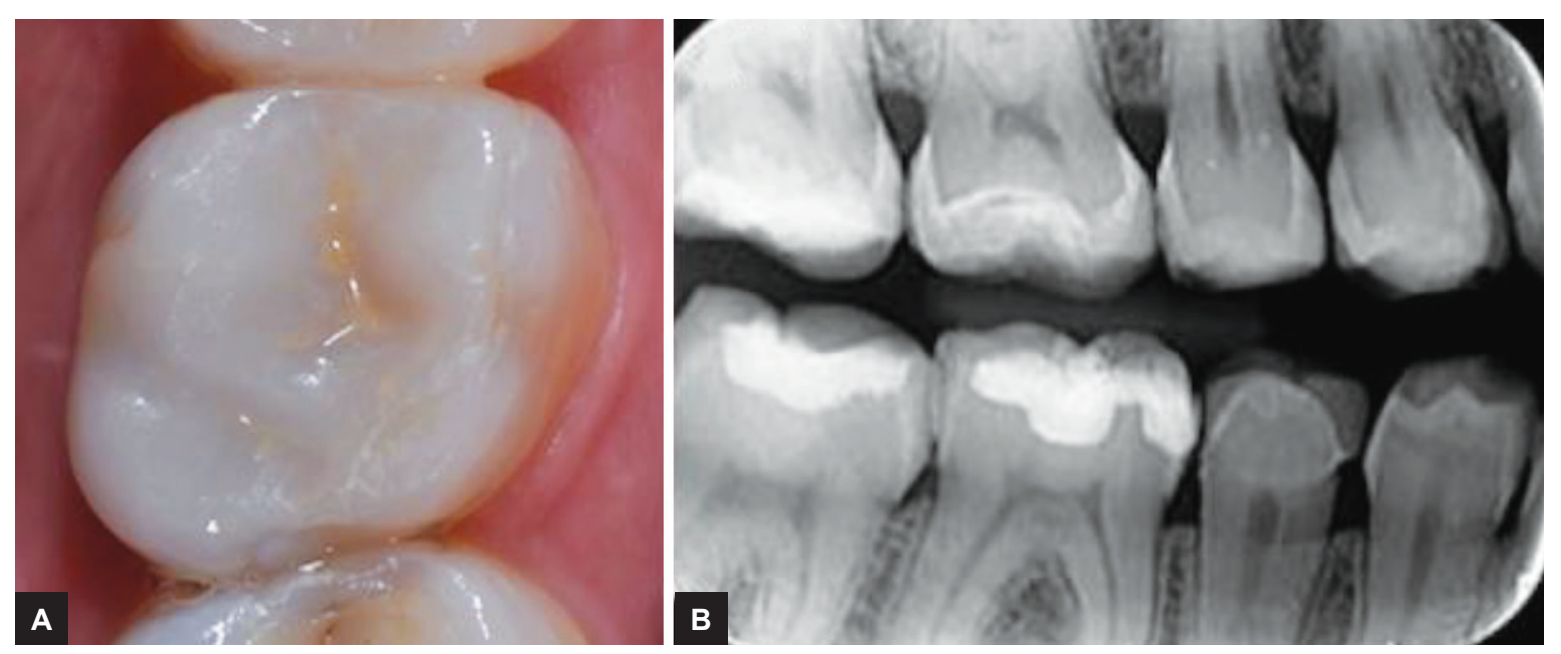

Figs 5A and B: (A) Clinical condition of the restored tooth after 15 months of follow-up; and (B) radiographic condition of the restored tooth after 15 months of follow-up

axis, and proximal contacts. Then, the sealed prepared tooth and the ceramic restoration were cleaned with a $70 \%$ isopropyl alcohol solution. After try-in, the intaglio surface of the final restoration was treated as follows:

- 10\% hydrofluoridric acid (Condac Porcelana, FGM, Joinville, Brazil) for 20 seconds as recommended by the manufacturer for lithium disilicate glass ceramic surface etching

- Air/water spray for 60 seconds

- $37 \%$ phosphoric acid (Condac 37, FGM) rubbed for 60 seconds with microbrush

- Air/water spray for 60 seconds

- Silane layer (Prosil, FGM)

- Uncured adhesive layer (Adhesive Adper Scotchbond Multi-Purpose, 3M ESPE, St. Paul, Minnesota, USA). The preparation surface treatment was performed as follows:

- $37 \%$ phosphoric acid (Condac 37, FGM) rubbed for 30 seconds on enamel margin and sealed dentin

- Air/water spray for 60 seconds and air dry for 20 seconds
- Uncured adhesive layer (Adhesive Adper Scotchbond Multi-Purpose, 3M ESPE).

The RelyX ARC (3M ESPE) resin cement was selected for restoration bonding. The preparation was filled with the cement and the restoration was then seated. The cement excesses were removed with a microbrush and final light curing was performed for 40 seconds per face (lingual, buccal, and occlusal). Final occlusal adjustment and polish were performed with ceramic polish system (Dhpro, Paranaguá, PR, Brazil) (Fig. 4C). The restoration was successful clinically and radiographically up to 15 months of follow-up presenting no alteration of color and shape and without marginal deterioration and painful symptomatology (Fig. 5).

\section{DISCUSSION}

Immediate dentin sealing has proved to be a reliable and predictable technique according to scientific evidence in the past years and may be an optimized approach whenever dentin is exposed during tooth preparation. ${ }^{1,9}$ 
Among the various advantages of using IDS, four can be outlined. First, the freshly cut dentin is the ideal dentin condition for bonding and this substrate is only achievable immediately after preparation. ${ }^{1,3,5,6}$ Second, the prepolymerization before cementation may interfere with restoration fitting, and thus should be avoided. On the contrary, IDS allows a polymerized resin coat that would not interfere on the restoration fitting. ${ }^{1,3,5,6}$ Third, the prepolymerization of the DBA before impression allows stress-free maturation of the hybrid layer, since bond strength develops progressively over time. ${ }^{1,6}$ Fourth, IDS protects dentin against bacterial infiltration during temporization, decreasing sensitivity. $1,5,6,10$

Some manufacturers recommend to keep the adhesive layer uncured before restoration of the final seating, though this approach may bring other problems. During restoration insertion, dentinal fluid may hydrolyze the DBA, incorporating micro-porosities within the hybrid layer. Hence, the pressure of the cement during the restoration seating may increase the collagen meshwork collapse. ${ }^{1,4-6}$

Many other clinical conditions support the various indications of IDS. Patient comfort is increased, since there is limited need for anesthesia during cementation, and also the resin coating decreases the postoperative sensitivity. It is also important to outline that the bonding procedure can be split into two different procedures: The wet bonding to dentin during IDS, and dry bonding of selectively etched enamel during final cementation. ${ }^{1}$

The two-step self-etch adhesive system consists in the application of a hydrophilic primer with acid monomers followed by the application of the hydrophobic adhesive. Since there is no prior acid etching, neither washing/drying of the substrate, the smear layer is not removed but incorporated into the hybrid layer. Since the demineralization of the dentin by the acid monomers and the infusion of the adhesive occur simultaneously, the demineralized dentin is more efficiently infused by the adhesive in a total-etch approach. ${ }^{10,16,17}$

For the total-etch approach, the acid etching of the dentin occurs before the primer infusion. Thus, the phosphoric acid removes the smear layer, opens the dentin tubules, demineralizes intertubular and peritubular dentin, increases the dentin permeability, and exposes the collagen meshwork. In this condition, the infusion of the hydrophilic primer is increased, which also facilitates the infusion of low-viscosity adhesive throughout the collagen meshwork into the dentin tubules, forming the hybrid layer. ${ }^{10,16,17}$

Magne et $\mathrm{al}^{5}$ stated that freshly cut dentin should be sealed immediately after tooth preparation, before impression or temporization. Either total-etch and selfetch adhesive systems are recommended by the authors.
It was also observed that even after 12 weeks of temporization onto the sealed dentin, the bond strength was maintained.

In the present case report, the self-etch technique was selected for its efficiency, technical simplicity, and the avoidance of applying phosphoric acid that could cause pulp inflammation. ${ }^{10,16,17}$

Simplified adhesive systems provide easier bonding protocols, as they reduce the amount of steps. It is also important because it decreases the technique sensitivity, as there is no need for etching with phosphoric acid before primer application. The risk of collagen degradation is also decreased since the demineralized dentin is almost fully infused by the adhesive. The prepolymerization of the DBA reduces the dentin permeability and decreases the bacterial penetration and dentin sensitivity in vital teeth. ${ }^{10,16,17}$

The adhesive system used in the presented clinical case (Clearfil SE Bond) contains methacryloyloxydecyl dihydrogen phosphate that provides chemical bonding to hydroxyapatite. It is widely used in dental research and the current study among self-etch systems. ${ }^{4,5}$ Choi and $\mathrm{Cho}^{4}$ performed an in vitro study testing the bond strength of IDS and delayed dentin sealing (DDS) in ceramic restorations. Two adhesive systems were compared: Clearfil SE Bond (Kuraray) and Adper Single Bond 2 (3M ESPE). The resin cement used was Variolink II (Ivoclar Vivadent, Schaan, Liechtentein). Immediate dentin sealing groups provided higher microtensile bond strength values than DDS groups for either adhesive and Clearfil SE Bond outperformed Adper Single Bond 2 within IDS groups.

Braz et $\mathrm{al}^{19}$ evaluated the bond strength to dentin of four different self-etch adhesive systems: Clearfil SE Bond (Kuraray), AdheSE (Ivoclar Vivadent, Schaan, Liechtenstein), Self-etch (Vigodent, Rio de Janeiro, Brazil), and Vitremer (3M ESPE) with different application techniques: Maintaining the smear layer on the substrate; removing the smear layer with $37 \%$ phosphoric acid for 15 seconds; and deproteinization of dentin (collagen removal) with $10 \%$ sodium hypochlorite for 10 seconds after phosphoric acid etching. The authors found that regardless the application protocol, Clearfil SE Bond provided the highest bond strength values, corroborating with the findings of Choi and Cho. ${ }^{4}$

The DBA may present an unpolymerized superficial layer up to $40 \mu \mathrm{m}$ thick due to the oxygen presence during light polymerization..$^{20}$ This unpolymerized layer may interfere with the impression material, such as polyvinyl siloxane and polyether. Thus, the clinical protocol has to include the application of the air-blocking layer of glycerin followed by a final 10 seconds of polymerization. ${ }^{20}$ 
Regarding the use of low-viscosity composite resin, Knobloch et $\mathrm{al}^{16}$ found that the additional composite resin coating over the adhesive layer did not increase bond strength. However, other studies suggest its use due to increased bond strength ${ }^{11,12,18}$ and inhibition of possible chemical incompatibility between IDS layer and resin cement. ${ }^{21}$

Helvey ${ }^{10}$ suggested that this clinical case was conducted with the combination of IDS with enamel etching at restoration delivery. After IDS, the enamel margins were finished and made free of adhesive excesses before impression. During restoration delivery, the whole preparation (sealed dentin and enamel) was etched with phosphoric acid to demineralize the enamel margin and also clean the resin coating layer of the sealed dentin. According to Magne et al, ${ }^{5}$ the IDS offers the clinician the opportunity to bond to dentin during the "wet bonding" phase and bond to enamel during the "dry bonding" phase at restoration delivery.

Whenever applying the IDS technique, there is no unanimous clinical protocol. There are many possibilities that vary according to different modifications on the technique and chosen materials. Due to the increased amount of DBA released into the market yearly, continuous research should be carried out to ensure that the various adhesive systems and resin cements are reliable for an evidence-based dental practicing.

\section{CONCLUSION}

Considering the reported case result and evidence in the current literature, IDS is expected to provide better bond strength, marginal fit, protection of dentin, and pulp throughout the temporary phase, and thus, is more long-lasting for the restoration and restored tooth. The patient reports comfort and lack of sensitivity during temporization, bonding, and follow-up of 15 months.

\section{REFERENCES}

1. Magne P. Immediate dentin sealing: a fundamental procedure for indirect bonded restorations. J Esthet Restor Dent 2005 May;17(3):144-154.

2. Sahin C, Cehreli ZC, Yenigul M, Dayangac B. In vitro permeability of etch-and-rinse and self-etch adhesives used for immediate dentin sealing. Dent Mater J 2012 May;31(3): 401-408.

3. de Andrade OS, de Goes MF, Montes MA. Marginal adaptation and microtensile bond strength of composite indirect restorations bonded to dentin treated with adhesive and low-viscosity composite. Dent Mater 2007 Mar;23(3):279-287.

4. Choi YS, Cho IH. An effect of immediate dentin sealing on the shear bond strength of resin cement to porcelain restoration. J Adv Prosthodont 2010 Jun;2(2):39-45.
5. Magne P, So WS, Cascione D. Immediate dentin sealing supports delayed restoration placement. J Prosthet Dent 2007 Sep;98(3):166-174.

6. Magne P, Kim TH, Cascione D, Donovan TE. Immediate dentin sealing improves bond strength of indirect restorations. J Prosthet Dent 2005 Dec;94(6):511-519.

7. $\mathrm{Hu}$ J, Zhu Q. Effect of immediate dentin sealing on preventive treatment for postcementation hypersensitivity. Int J Prosthodont 2010 Jan-Feb;23(1):49-52.

8. Dalby R, Ellakwa A, Millar B, Martin FE. Influence of immediate dentin sealing on the shear bond strength of pressed ceramic luted to dentin with self-etch resin cement. Int J Dent 2012;2012:310702.

9. Qanungo A, Aras MA, Chitre V, Mysore A, Amin B, Daswani SR. Immediate dentin sealing for indirect bonded restorations. J Prosthodont Res 2016 Oct;60(4):240-249.

10. Helvey GA. Adhesive dentistry: the development of immediate dentin sealing/selective etching bonding technique. Compend Contin Educ Dent 2011 Nov-Dec;32(9):22, 24-32, 34-35.

11. Jayasooriya PR, Pereira PN, Nikaido T, Tagami J. Efficacy of a resin coating on bond strengths of resin cement to dentin. J Esthet Restor Dent 2003 Mar;15(2):105-113.

12. Santos-Daroz CB, Oliveira MT, Góes MF, Nikaido T, Tagami J, Giannini M. Bond strength of a resin cement to dentin using the resin coating technique. Braz Oral Res 2008 JulSep;22(3):198-204.

13. Giannini M, Takagaki T, Bacelar-Sá R, Vermelho PM, Ambrosano GM, Sadr A, Nikaido T, Tagami J. Influence of resin coating on bond strength of self-adhesive resin cements to dentin. Dent Mater J 2015 Dec;34(6):822-827.

14. Brigagão VC, Barreto LFD, Gonçalves KAS, Amaral M, Vitti RP, Neves ACC, Silva-Concilio LR. Effect of interim cement application on bond strength between resin cements and dentin: Immediate and delayed dentin sealing. J Prosthet Dent 2017 Jun;117(6):792-798.

15. Ishii N, Maseki T, Nara Y. Bonding state of metal-free CAD/ CAM onlay restoration after cyclic loading with and without immediate dentin sealing. Dent Mater J 2017 May;36(3): 357-367.

16. Knobloch LA, Gailey D, Azer S, Johnston WM, Clelland N, Kerby RE. Bond strengths of one- and two-step self-etch adhesive systems. J Prosthet Dent 2007 Apr;97(4):216-222.

17. Grégoire G, Guignes P, Millas A. Effect of self-etching adhesives on dentin permeability in a fluid flow model. J Prosthet Dent 2005 Jan;93(1):56-63.

18. Okuda M, Nikaido T, Maruoka R, Foxton RM, Tagami J. Microtensile bond strengths to cavity floor dentin in indirect composite restorations using resin coating. J Esthet Restor Dent 2007 Jan-Feb;19(1):38-46.

19. Braz R, Ribeiro AI, Dantas DC, Correia TC, Figueiroa AF, Cavalcanti AL. Adesivos autocondicionantes: efeito do condicionamento ácido e proteolítico na resistência de união. Pesqui Bras Odontopediatria Clin Integr 2011 Jan-Mar;11(1):41-46.

20. Magne P, Nielsen B. Interactions between impression materials and immediate dentin sealing. J Prosthet Dent 2009 Nov;102(5):298-305.

21. Rathke A, Balz U, Muche R, Haller B. Effects of self-curing activator and curing protocol on the bond strength of composite core buildups. J Adhes Dent 2012 Feb;14(1):39-46. 